\title{
Teoria dos jogos aplicada à ecologia matemática
}

\author{
Augusto César de Castro Barbosa, Carlos Antônio de Moura, \\ Instituto de Matemática e Estatística, IME, UERJ, \\ 20550-900, Rio de Janeiro, RJ \\ E-mail: accb@ime.uerj.br, demoura@ime.uerj.br, \\ Daniela Ribeiro Monteiro \\ Depto de Engenharia Mecânica, FEN, UERJ \\ 20940-903, Rio de Janeiro, RJ \\ E-mail: danielarmonteiro@gmail.com.
}

\begin{abstract}
Resumo: A teoria dos jogos é utilizada junto à teoria do controle para modelar matematicamente um problema em ecologia: o controle de pragas em uma dada lavoura. Trata-se de um problema relevante e muito investigado, com diferentes ferramentas - em particular com aquelas de controle ótimo.
\end{abstract}

Palavras-chave: Teoria dos jogos, controle ótimo, sistemas biológicos

\section{Introdução}

A ecologia é a ciência que pesquisa as interações entre os organismos e seu ambiente. As interações podem ser entre seres vivos e/ou com o meio ambiente. A ecologia matemática se propõe a utilizar modelos matemáticos para analisar, entender, avaliar, prever fenômenos ecológicos. Neste trabalho apresentamos um problema real na agricultura: o controle de pragas. Para realizar esse controle, utilizamos as equações de Lotka-Volterra [2, 9] com competição. Introduzimos um controle e, com o emprego de dados reais, montamos um jogo para o qual obtemos um equilíbrio de Nash. Trata-se de uma aplicação da teoria dos jogos diferenciáveis, uma área que une a teoria dos jogos (clássica) à teoria de controle.

Thomas Malthus (1766-1834) foi o responsável pela teoria populacional denominada malthusiana. Malthus observou que o crescimento populacional, entre 1650 e 1750, dobrou como consequência do aumento da produção de alimentos, das melhorias das condições de vida nas cidades, do aperfeiçoamento do combate às doenças, das melhorias no saneamento básico e dos benefícios obtidos com a revolução industrial. Esses fatores provocaram um declínio na taxa de mortalidade, ampliando o crescimento natural. Preocupado com o crescimento populacional acelerado, Malthus publicou em 1798 uma série de ideias alertando para a importância do controle da natalidade.

\section{Teoria dos Jogos}

A teoria dos jogos é o estudo matemático do contexto das tomadas de decisão entre indivíduos, sendo a escolha de cada um dependente das decisões dos demais. Essa teoria foi inicialmente formalizada com a publicação por John von Neumann e Oskar Morgenstern do texto The theory of games and economic behavior [5]. Os elementos básicos de um jogo são: os jogadores, as regras do jogo, os resultados e os ganhos.

Os jogos são classificados quanto ao número de jogadores: um jogador, dois jogadores, $n$ jogadores e quanto ao seu tipo, podendo ser: com ou sem repetição, finito, de soma zero ou 
soma não zero, simultâneos ou sequenciais, informação perfeita ou imperfeita e cooperativo ou não cooperativo.

Introduzimos a proposta de um jogo diferenciável no controle de pragas no campo. Para isso, determinaremos os jogadores, o plano de fundo e as condições do jogo.

O jogo é constituído por dois jogadores, sendo eles: Jogador I - as pragas (neste caso, Lagarta da Soja) e Jogador II - o agricultor (representado pelos inimigos naturais da lagarta da soja).

O controle de pragas provoca benefícios e malefícios. Manter uma plantação sem o uso de agrotóxico a torna muito mais vulnerável à ação das pragas, o que induz à perda da produção. Consequentemente, aumenta o valor final do produto, tendo em vista uma menor produção por área comparativamente àquela obtida com emprego de agrotóxico.

O plano de fundo do jogo será uma lavoura infestada por uma certa praga, cujo controle biológico será um inimigo natural da praga.

\section{Modelagem Matemática de dinâmica populacional}

Um sistema de equações diferenciais ordinárias (EDO) apresenta a forma

$$
d x / d t=g(t, x)
$$

onde a lei $g$, que regula a taxa de variação da variável $x$ com relação ao tempo, depende não somente de $x$ (eventualmente multidimensional), mas também do tempo $t$, variável unidimensional. O sistema apresentado é chamado de sistema não-autônomo. Já um sistema autônomo é aquele cuja configuração independe do tempo. Assim podemos sempre considerar o instante inicial como sendo $t=0$.

Malthus em [3] conjecturou que, dados recursos abundantes, as populações humanas apresentam a tendência de crescimento geométrico. Esse padrão pode ser descrito com:

$$
d x / d t=x r
$$

onde $r(r>0)$ é a taxa de crescimento relativo da população $x$.

Em 1838 Verhulst [8] propôs uma modificação na equação de Malthus onde considera que os recursos são limitados, e a taxa de crescimento da população, proporcional à população em cada instante, criando a equação logística para descrever o crescimento de uma população com recursos limitados. A equação logística é normalmente escrita como uma equação diferencial do tipo:

$$
d x / d t=x(a-\gamma x),
$$

onde $d x / d t$ é a taxa de crescimento populacional, $x=x(t)$ é a densidade da população em um instante $t, a$ é uma constante positiva que representa a taxa de crescimento populacional quando não há fatores limitantes e $\gamma$ é uma constante positiva que representa o limite populacional, isto é, o valor para o qual a população tende no decorrer do tempo.

A equação logística considera que a taxa de crescimento, $d x / d t$, depende não só da população, mas também das condições existentes no meio em que está presente. $\mathrm{O}$ termo $(-\gamma x) x$ na equação representa a contribuição da competição entre os indivíduos da mesma espécie no crescimento da população. Esta equação retrata uma única população.

O modelo de Lotka-Volterra foi pioneiro ao descrever matematicamente a interação entre duas populações distintas (presas e predadores). A introdução deste modelo, bem como as suas consequentes variações, foi uma das principais contribuições para a dinâmica de populações. A relação entre presa e predador é descrita da seguinte forma:

$$
\left\{\begin{array}{l}
d x / d t=x(a-\alpha y) \\
d y / d t=y(-b+\beta x)
\end{array} .\right.
$$


As equações, chamadas equações Lotka-Volterra, onde $x=x(t)$ e $y=y(t)$ representam, respectivamente, as populações de presas/hospedeiro e predadores/parasitóide, foram construídas a partir das seguintes hipóteses:

1. $d x / d t=a x, \operatorname{com} a>0$ quando $y=0$

2. $d y / d t=-b y$, com $b>0$ quando $x=0$

3. Os termos $\beta x y$ e $-\alpha y x, \beta>0$ e $\alpha>0$, representam as interações entre as duas espécies. O termo $\beta x y$ implica no crescimento de $y$, enquanto o termo - $\alpha y x$ implica no decrescimento de $x$.

Supõe-se que os recursos para as presas são ilimitados, e que o único fator inibidor para seu crescimento é a presença dos predadores.

Trataremos agora de um modelo matemático que une os dois modelos acima, isto é, o modelo da equação logística e o modelo de Lotka-Volterra. Vamos admitir na primeira equação de (3), o fator natural que inibe o crescimento da população, isto é, essa equação é modificada para, quando $y$ for igual a zero, obtermos (2). O sistema é definido como um caso particular de (3), sendo referido como modelo de Lotka-Volterra com competição,

$$
\left\{\begin{array}{l}
d x / d t=x(a-\gamma x-\alpha y) \\
d y / d t=y(-b+\beta x)
\end{array},\right.
$$

onde:

a. $a, \gamma, \alpha, b$ e $\beta$ são constantes positivas;

b. $a$ representa a taxa de crescimento das pragas;

c. $b$ representa a taxa de mortalidade dos inimigos naturais das pragas;

d. $-\alpha y x$ e $\beta x y$ são as interações entre as duas populações;

e. $(-\gamma x) x$ representa a competição intraespecífica das pragas, que concorrem pelo alimento em lavouras.

Esse modelo considera a existência de um número elevado de presas, não sendo portanto o foco da análise a competição intraespecífica entre predadores. Assim, não introduzimos um termo que corresponderia à competição intraespecífica na equação dos predadores.

Considerando o sistema (4), podemos calcular seus pontos de equilíbrio:

$$
\left\{\begin{array}{l}
x(a-\gamma x-\alpha y)=0 \\
y(-b+\beta x)=0
\end{array} .\right.
$$

Como as condições $x=0$ ou $y=0$ não apresentam interesse para o modelo, pois $x$ e $y$ representam as densidades das populações. Logo, o ponto de equilíbrio para o sistema sem controle é

$$
\left(\frac{b}{\beta}, \frac{a-\frac{\gamma b}{\beta}}{\alpha}\right) \text {. }
$$

O ponto de equilíbrio do sistema (4) possui um nível maior do que o desejável, mesmo o modelo sendo estável. Por isso, neste caso, é necessário a aplicação do controle.

Observe que o número de pragas no ponto de equilíbrio é invariante relativamente aos coeficientes da primeira equação. Ou seja, mudar qualquer parâmetro na primeira equação não auxiliaria para reduzir essa população.

De fato, teríamos

$$
\left\{\begin{array}{l}
d x / d t=x\left(a a_{1}-\gamma \gamma_{1} x-\alpha \alpha_{1} y\right) \\
d y / d t=y(-b+\beta x)
\end{array},\right.
$$

cujo ponto de equilíbrio para o sistema sem controle é

$$
\left(\frac{b}{\beta}, \frac{a a_{1}-\frac{\gamma \gamma_{1} b}{\beta}}{\alpha \alpha_{1}}\right) \text {. }
$$


Já para a segunda equação, alterar os valores dos coeficientes implica na obtenção de valores diferentes para as pragas no equilíbrio:

$$
\left\{\begin{array}{l}
d x / d t=x(a-\gamma x-\alpha y) \\
d y / d t=y\left(-b b_{1}+\beta \beta_{1} x\right)
\end{array}\right.
$$

cujo ponto de equilíbrio é

$$
\left(\frac{b b_{1}}{\beta \beta_{1}}, \frac{a-\frac{\gamma b b_{1}}{\beta \beta_{1}}}{\alpha}\right) .
$$

Essa expressão significa que obtivemos:

$i$. Aumento do número de pragas caso $b_{1}>\beta_{1}$

ii. Diminuição do número de pragas caso $b_{1}<\beta_{1}$

iii. Mesmo número de pragas caso $b_{1}=\beta_{1}$

Para a estratégia de controle biológico, onde é feita a inserção de inimigos naturais, de modo a manter o sistema em um estado de equilíbrio abaixo de danos econômicos, utilizamos o modelo (4), introduzindo na segunda equação uma função de controle, denominada $U$. Assim, a equação que descreve a taxa de variação da densidade populacional do inimigo natural da praga em questão é

$$
\left\{\begin{array}{l}
d x / d t=x(a-\gamma x-\alpha y) \\
d y / d t=y(-b+\beta x)+U
\end{array} .\right.
$$

A aplicação de uma função de controle se baseia na ideia de que existe um certo limite de quantidade de pragas que determina a margem de danos econômicos, denotada por $x_{d}$.

\section{Aplicação ao controle de pragas em lavouras de soja}

A lagarta-da-soja é encontrada em todos os locais de cultivo, sendo o desfolhador mais comum da soja no Brasil. Costuma atacar as lavouras a partir de novembro, nas regiões ao norte do Paraná, e a partir de dezembro a janeiro no sul do país, podendo causar desfolhamento que pode chegar até a 100\%. Segundo os níveis de ação pelo MIP-Soja [1], A. gemmatalis deve ser controlada com inseticidas químicos, quando forem encontradas, em média, 20 lagartas pequenas (igual ou superior a $1.5 \mathrm{~cm}$ ) por metro quadrado. No caso de ataques da lagarta-da-soja, existem várias opções de produtos eficientes, tanto os inseticidas químicos quanto os biológicos, a exemplo do Baculovirus anticarsia, específico para a lagarta-da-soja.

O agricultor busca diminuir a quantidade de lagartas por hectare com o auxílio de inimigos naturais no meio ambiente. Para isso, ele busca encontrar um controle $U$ de modo que a quantidade total de lagartas permaneça num patamar economicamente estável. Usaremos $x_{d}=$ 20 (vinte lagartas pequenas) como ponto de equilíbrio estável para o número de lagartas.

Voltando às equações de Lotka-Volterra com competição

$$
\left\{\begin{array}{l}
d x / d t=x(a-\gamma x-\alpha y) \\
d y / d t=y(-b+\beta x)+U
\end{array},\right.
$$

desejamos encontrar $U$ de modo que o ponto $P=\left(x_{d}, y_{d}\right)$ seja economicamente estável.

Utilizaremos no nosso jogo os dados de Santos [6] para os parâmetros das equações acima. 
Tabela 1: Dados da EMBRAPA.

\begin{tabular}{|c|c|c|c|c|}
\hline$a$ & $b$ & $\alpha$ & $\gamma$ & $\beta$ \\
\hline 0.16 & 0.19 & 0.02 & 0.001 & 0.0029 \\
\hline
\end{tabular}

Substituindo os valores dos parâmetros nas equações sem controle obtemos:

$$
\left\{\begin{array}{l}
d x / d t=x(0.16-0.001 x-0.02 y) \\
d y / d t=y(-0.19+0.0029 x)
\end{array} .\right.
$$

Fazendo

$$
\left\{\begin{array}{l}
x(0.16-0.001 x-0.02 y)=0 \\
y(-0.19+0.0029 x)=0
\end{array},\right.
$$

obtemos como ponto de equilíbrio o valor $P_{i} \cong(65.5 ; 4.7)$, um valor que não é economicamente viável para o agricultor.

Substituindo $x=x_{d}=20$ em $(0.16-0.001 x-0.02 y)=0$, obtemos $y_{d}=7$, ou seja, $P_{f}=\left(x_{d}, y_{d}\right)=(20,7)$.

O agricultor busca então sair do ponto $P_{i} \cong(65.5 ; 4.7)$ para o ponto $P_{f}=\left(x_{d}, y_{d}\right)=(20,7)$. Para isso ele utilizará um controle $U$ na segunda equação, ou seja:

$$
\left\{\begin{array}{l}
d x / d t=x(0.16-0.001 x-0.02 y) \\
d y / d t=y(-0.19+0.0029 x)+U
\end{array} .\right.
$$

Variando gradualmente o parâmetro $b$ da equação (4), observamos que, quando $b=0.058$, a solução do sistema é o ponto $(20,7)$, ou seja, o ponto economicamente estável. Assim sendo, determinamos $U=0.132 y$ e podemos reescrever o sistema conforme segue:

$$
\left\{\begin{array}{l}
d x / d t=x(0.16-0.001 x-0.02 y) \\
d y / d t=y(-0.19+0.0029 x)+0.132 y
\end{array} .\right.
$$

Determinando para cada valor de

$$
b \in\{x \in \mathbb{R} \mid 0.058 \leq x \leq 0.019\}
$$

o ponto

$$
P^{*}=\left(\frac{b}{0,0029}, \frac{0.16-0.001 \frac{b}{0.0029}}{0.02}\right),
$$

obtemos o gráfico que segue.

Figura 1: - Variação do parâmetro

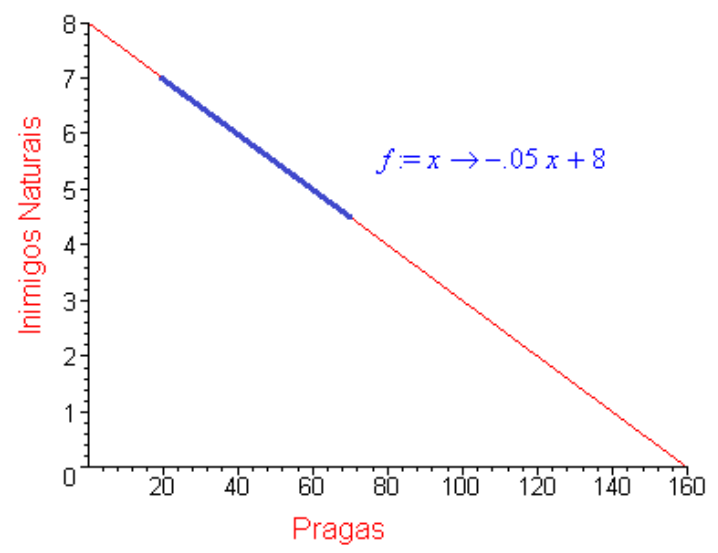


Variando a função até os pontos (0.8) e (160.0), obtemos a função linear $f(x)=-0.05 x+8$. A partir dessa função, podemos montar a seguinte tabela.

Figura 2: - Jogo lagarta $\mathrm{X}$ inimigos naturais

\begin{tabular}{|c|c|c|}
\hline \multicolumn{1}{c|}{} & \multicolumn{2}{c|}{ Lagartas } \\
\hline Inimigos & Livres no Ambiente & Fora do Ambiente \\
\hline Livres no Ambiente & $7 ; 20$ & $8 ; 0$ \\
\hline Fora do Ambiente & $0 ; 160$ & $0 ; 0$ \\
\hline
\end{tabular}

Esta tabela é a representação do nosso jogo. Observe que dadas as equações de Lotka-Volterra com competição e realizando uma variação gradual no parâmetro $b$ para assim conseguir obter o controle $U$, montamos o gráfico acima. Este gráfico nos forneceu a tabela que simboliza um jogo.

A partir dessa tabela, verificamos que o ponto $(20,7)$ é um equilíbrio de Nash, não havendo nenhum outro nesse jogo.

Figura 3: - Equilíbrio de Nash

\begin{tabular}{|l|c|c|}
\cline { 2 - 3 } \multicolumn{1}{c|}{} & \multicolumn{2}{|c|}{ Lagartas } \\
\hline Inimigos & Livres no Ambiente & Fora do Ambiente \\
\hline Livres no Ambiente & $7 ; 20$ & $8 ; 0$ \\
\hline Fora do Ambiente & $0 ; 160 \leftarrow$ & $0 ; 0$ \\
\hline
\end{tabular}

Concluímos então que para ir do ponto $P_{i} \cong(65.5 ; 4.7)$ para o ponto $P_{f}=\left(x_{d}, y_{d}\right)=(20,7)$, o agricultor deve modificar o parâmetro referente à taxa de mortalidade dos inimigos naturais. Para isso, basta inserir no meio maior quantidade de inimigos, fazendo com que essa taxa decresça e se consiga assim atingir o ponto economicamente estável. 


\section{Referências}

[1] Embrapa, "Soja - Manejo integrado de pragas", Circular Técnica, Londrina, 2000.

[2] A.J.Lotka, "Elements of physical biology", Williams \& Wilkins Company, Baltimore, 1925.

[3] R.T. Malthus, "An essay on the principle of population", J. Johnson, St. Paul's ChurchYard, 1798.

[4] D.R. Monteiro, "Uma introdução à teoria dos jogos", Monografia, UERJ, Rio de Janeiro, 2010 .

[5] J.V. Neumann, O. Morgenstern, "Theory of games and economic behavior", Princeton University Press, New Jersey, 1944.

[6] L.H. Santos, "Teoria de controle ótimo com aplicações a sistemas biológicos", Dissertação, UERJ, Rio de Janeiro, 2012.

[7] M. Rafikov, "Notas do minicurso: aplicação dos modelos matemáticos no controle de populações", UFSC, Santa Catarina, 2003.

[8] P.F. Verhulst, "Notice sur la loi que la population poursuit dans son accroissement", Correspondance mathématique et physique, 1838.

[9] V. Volterra, "Fluctuations in the abundance of a species considered mathematically", Nature, 1926. 